\title{
Analysis of Standing Waves in Millimetre-Wave Optics
}

\author{
M. Whale ${ }^{(l)}$, N Trappe ${ }^{(l)}$, J.A. Murphy ${ }^{(l)}$, Member, IEEE S. Withington ${ }^{(2)}$, Member, IEEE \\ ${ }^{(1)}$ National University of Ireland, Maynooth, Co.Kildare. Ireland. \\ ${ }^{(2)}$ Cavendish Laboratory, Madingley Road, Cambridge CB3 0HE. UK
}

\begin{abstract}
In this paper, we expand a theoretical framework for modelling standing waves in millimeter/submillimetre optical systems. Standing waves or multiple reflections have been traditionally difficult to model but this analytical method proves to be very versatile in dealing with reflections between typical quasioptical components such as lenses, apertures and mirrors. Using a full multi moded description of the feed horn (typically a corrugate horn), which is then transformed to equivalent free space Gaussian modes, a full mode matching scattering matrix approach is utilized to analyze any optical path/element, in which track is kept of both the backward and forward propagating components of the fields. We present an analysis of the reflected power from complicated cascaded optical apertures in a situation similar to a stray light baffle. Interesting constructive and destructive interference conditions can be observed from these structures and can be uniquely modelled with the presented theoretical technique.
\end{abstract}

Index Terms-Gaussian beam mode analysis, scattering matrix, quasioptical system, standing waves.

\section{INTRODUCTION}

$\mathrm{T}$ HE now standard theoretical framework for quasioptical beam guide systems based on Gaussian beam mode analysis [1] can be successfully extended to include partial reflections and standing waves in $\mathrm{THz}$ optical systems fed by horn antennas. Propagation in the uniform waveguide and beamguide sections of the system can be accurately described in terms of the corresponding modes, with the effects of any discontinuities modelled using a mode matching approach [2]. By combining free space propagation using Gaussian beam mode analysis [3] with waveguide modes in the horn, complete optical systems can be analyzed [4]. In the modal view of propagation, waveguide modes are transformed at the horn aperture to free space Gaussian modes that subsequently propagate through the optics. Both because of truncation and aberrational effects, realistic optical components disturb the modal propagation. At such optical components power is scattered between modes. These components are broken down into a combination of standard elements, each with its own specific scattering matrix. These scattering matrices are then cascaded together to form a complete description of the optical system. Similarly, an optical system can be divided into a sequential arrangement of these optical elements, which can be cascaded together to give the complete scattering matrix description of the optical path. The effect of this system on the beam can be described in terms of transmission and reflection coefficients for the forward and backward propagating modes. This method forms the basis of a theoretical method to analyse multiple reflections.

\section{THEORY}

The mode matching technique can be regarded as a method for obtaining the overall transmission and reflection of any optical path element, or the optical system as a whole. The optical system is comprised of a sequential series of optical components, each with a scattering matrix which describes the distribution of power carried by the incident field between the adjacent modes. A set of column matrices represents the mode coefficients of the forward and backward propagating modes.

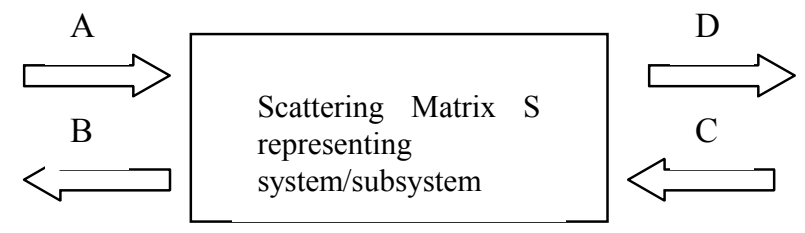

Fig. 1. The scattering matrix $S$ describes a modular section of a quasi-optical system and relates the coefficients on the input side (left) to the coefficients on the output side (right hand side)

We let the matrices [A] and [B] represent the incident and reflected mode coefficients as viewed from the input port of the system, and the matrices [C] and [D] represent the incident wave from the right (output) side of the system and the transmission coefficients respectively. The mode coefficient are related through the following equation [1]

$$
\left[\begin{array}{l}
{[B]} \\
{[D]}
\end{array}\right]=[S]\left[\begin{array}{l}
{[A]} \\
{[C]}
\end{array}\right]
$$

where $[\mathrm{S}]$ is the scattering matrix describing the system., which is itself comprised of sub matrix components

$$
[S]=\left[\begin{array}{ll}
{\left[S_{11}\right]} & {\left[S_{12}\right]} \\
{\left[S_{21}\right]} & {\left[S_{22}\right]}
\end{array}\right]
$$

Theoretically, the dimensions of these matrices can run to infinity, but for computational practicality we limit them to a finite size. Combining equations (1) and (2), we get

$$
\left[\begin{array}{l}
{[B]} \\
{[D]}
\end{array}\right]=\left[\begin{array}{l}
{\left[S_{11}\right][A]+\left[S_{12}\right][C]} \\
{\left[S_{21}\right][A]+\left[S_{22}\right][C]}
\end{array}\right]
$$


It is a reasonable assumption that there will be no incident field towards the output port of the system, essentially making the coefficient set $[\mathrm{C}]$ equal to zero.

$$
[B]=\left[S_{11}\right][A], \quad[D]=\left[S_{21}\right][A]
$$

The input mode coefficients describing the feed horn [A] are written as a column matrix of finite size corresponding to the number of modes used.

Scattering matrices describing sequential elements in the optical path are cascaded together in the following manner. Let $\left[\mathrm{S}^{\mathrm{a}}\right]$ and $\left[\mathrm{S}^{\mathrm{b}}\right]$ be two such scattering matrices,

$$
\left[S^{a}\right]=\left[\begin{array}{ll}
{\left[S_{11}^{a}\right]} & {\left[S_{12}^{a}\right]} \\
{\left[S_{21}^{a}\right]} & {\left[S_{22}^{a}\right]}
\end{array}\right] \quad\left[S^{b}\right]=\left[\begin{array}{ll}
{\left[S_{11}^{b}\right]} & {\left[S_{12}^{b}\right.} \\
{\left[S_{21}^{b}\right]} & {\left[S_{22}^{b}\right.}
\end{array}\right]
$$

and the cascaded matrix is $\left[\mathrm{S}^{\mathrm{c}}\right]$,

$$
\left[S^{c}\right]=\left[\begin{array}{ll}
S_{11}^{c} & S_{12}^{c} \\
S_{21}^{c} & S_{22}^{c}
\end{array}\right]
$$

The sub matrices of $\left[\mathrm{S}^{\mathrm{c}}\right]$ are given by [5]

$$
\begin{aligned}
& {\left[S_{11}^{c}\right]=\left[S_{12}^{a}\right]\left[[I]-\left[S_{11}^{b}\right]\left[S_{22}^{a}\right]\right]^{-1}\left[S_{11}^{b}\right]\left[S_{21}^{a}\right]+\left[S_{11}^{a}\right]} \\
& {\left[S_{12}^{c}\right]=\left[S_{12}^{a}\right]\left[[I]-\left[S_{11}^{b}\right]\left[S_{22}^{a}\right]\right]^{-1}\left[S_{12}^{b}\right]} \\
& {\left[S_{21}^{c}\right]=\left[S_{21}^{b}\right]\left[[I]-\left[S_{22}^{a}\right]\left[S_{11}^{b}\right]\right]^{-1}\left[S_{21}^{a}\right]} \\
& {\left[S_{22}^{c}\right]=\left[S_{21}^{b}\right]\left[[I]-\left[S_{22}^{a}\right]\left[S_{11}^{b}\right]\right]^{-1}\left[S_{22}^{a}\right]\left[S_{12}^{b}\right]+\left[S_{22}^{b}\right]}
\end{aligned}
$$

This cascade matrix approach can be applied to the entire optical system, giving a complete modal description of the field at the output of the system.

\section{SYSTEMS ANALYSED}

In previous papers analysis of various common optical components used in quasioptical systems such as circular apertures, central blockages and classical examples such as a Fabry-Perot interferometer were investigated. We also have illustrated an experimental verification in a simple optical system [4].

In this paper we apply the theory to a more complicated optical system such as a stray light baffle as illustrated in figure 2. We model a stray light baffle as a series of annular rings and using equation 7 above apply the scattering theory to obtain an overall reflection and transmission profile for such a structure. Interesting interference effects dependent on the relative path length between the individual rings can be demonstrated. Figure 3 shows the reflected power in terms of both total power and also power contained in the fundamental mode as compared to the reflected power $\left(\mathrm{S}_{11}\right)$ with the corrugated horn structure itself.



Fig. 2. A series of annular rings in series are used in the model to represent a stay light baffle pipe. A full reflection and transmission analysis can be carried out on each of the rings in turn to obtain an overall reflection characteristic.

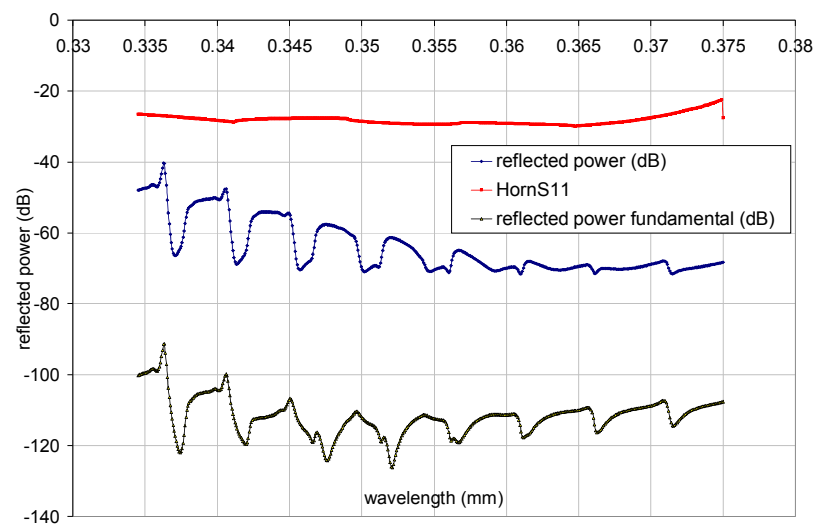

Fig. 3. The reflected power from a baffle containing six rings of 2 incident beam radii. The S11 from the horn itself is shown together with the power contained in the equivalent fundamental Gaussian and the total power. By varying the inter ring distance relative interference effects are also observed.

\section{CONCLUSIONS}

In this paper the developed code based on Gaussian Beam Mode Analysis to model multiple reflections or standing waves is extended to model complicated structures such as a cascaded series of circular apertures similar to the structure of a stray light baffle often used in optical systems.

\section{ACKNOWLEDGMENT}

The author of this paper would like to acknowledge the financial support of Science Foundation Ireland (SFI) through a Research Frontiers Grant for this research.

\section{REFERENCES}

[1] R. Padman and J.A. Murphy, "A Scattering matrix formulation for Gaussian Beam mode analysis" in Proc. Inst. Elct. Eng. $7^{\text {th }}$ Int. Conf. Antennas and Propagation (ICAP '91), Apr. 1991, pp. 201 - 204

[2] A.D. Olver, P.J.B. Clarricoats, A.A. Kishk and L. Shafai, Microwave Horns and Feeds. Piscataway, NJ: IEEE Press, 1994

[3] P. Goldsmith, Quasioptical Systems: Gaussian Beam Quasioptical Propagation and Applications. Piscataway, NJ: IEEE Press, 1998

[4] J.A. Murphy, N. Trappe, and S. Withington ,"Gaussian beam mode analysis of partial reflections in simple quasioptical system fed by horn antennas", Infrared Phys. Technol., vol. 44, no. 4, Aug. 2003, pp $289-$ 287

[5] N. Trappe, J.A. Murphy, S. Withington and W. Jellema, "Gaussian beam mode analysis of standing waves between two coupled corrugated horns", IEEE Trans. Antennas Propag., vol. 53. no. 5, May 2005 